\title{
1,3,4-Oxadiazole as a Potential Anti-Cancer Scaffold: A Review
}

\author{
Mohammad Mahboob Alam 1,* iD \\ 1 Department of Chemistry, Faculty of Science, Albaha University, Albaha, Kingdom of Saudi Arabia \\ * Correspondence: mmalamchem81@gmail.com; malam@bu.edu.sa;
}

Scopus Author ID 54941338500

Received: 1.08.2021; Revised: 8.09.2021; Accepted: 10.09.2021; Published: 21.10.2021

\begin{abstract}
N}=\mathrm{C}=\mathrm{O}$ - linkage. 1,3,4-oxadiazole derivatives possess remarkable biological properties; antimicrobial anti-inflammatory, anti-cancer, antitubercular, antioxidant, antiviral, and anti-diabetic. This scaffold is present in many marketed drugs, such as Raltegravir, Tiodazosin, Nesapidil, and Zibotentan. 1,3,4-oxadiazole derivatives have displayed significant anti-cancer potential with a diverse mode of actions viz. growth factors, enzymes, kinases, etc. The present review gives an overview of the anti-cancer potential of 1,3,4-oxadiazoles derivatives in cancer drug discovery and development from the last ten years.
\end{abstract}

Keywords: 1,3,4-oxadiazole; anti-cancer; heterocycle.

(c) 2021 by the authors. This article is an open-access article distributed under the terms and conditions of the Creative Commons Attribution (CC BY) license (https://creativecommons.org/licenses/by/4.0/).

\section{Introduction}

Heterocycles play a very important role in medicinal chemistry. The heterocycles have significant biological activity, due to which they are continued explored [1-5]. 1,3,4oxadiazole is an aromatic heterocycle with $-\mathrm{N}=\mathrm{C}=\mathrm{O}$ - linkage. It contains one oxygen and two nitrogen atoms and is found in different isomeric forms (Figure 1).

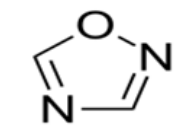

1,2,4-oxadiazole<smiles>c1nnco1</smiles>

1,3,4-oxadiazole<smiles>c1cnon1</smiles>

1,2,5-oxadiazole<smiles>c1conn1</smiles>

1,2,3-oxadiazole

Figure 1. Different isomers of oxadiazole.

1,3,4-oxadiazole derivatives possess remarkable biological properties. It can be used as antimicrobial [6-11], anti-inflammatory [12-14], anticancer [15-18], antioxidant [19], antiviral [20, 21], antidiabetic [22, 23]. Oxadiazole derivatives can be found in marketed drugs such as Raltegravir - an antiretroviral drug against HIV, Ataluren - in treating cystic fibrosis, Tiodazosin, and Nesapidil used as antihypertensive drugs, Zabotentan - in the final stage of clinical trials as anti-cancer agents (Figure 2). Besides these, 1,3,4-oxadiazole also finds applications in material science [24-26].

1,3,4-oxadiazole derivatives have displayed significant anti-cancer potential with the diverse mode of actions such as growth factors, enzymes, kinases, etc. [27]. This review article gives an overview of the anti-cancer potential of 1,3,4-oxadiazoles in cancer drug discovery and development from the last ten years. 


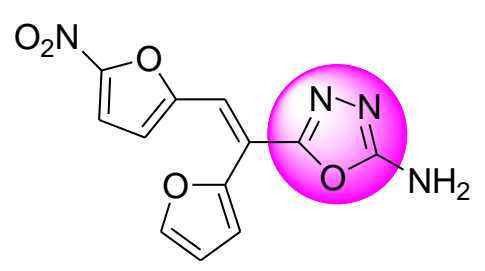

Furamizole (Antibiotic)<smiles>COc1ccccc1N1CCN(CC(C)COc2cccc(-c3nnc(C)o3)c2)CC1</smiles>

Nesipidil

(Antihypertensive drug)<smiles>COc1nccnc1NS(=O)(=O)c1cccnc1-c1ccc(C23CC2CCCCCCCCCN3)cc1</smiles>

Zibotentan (Anticancer drug)

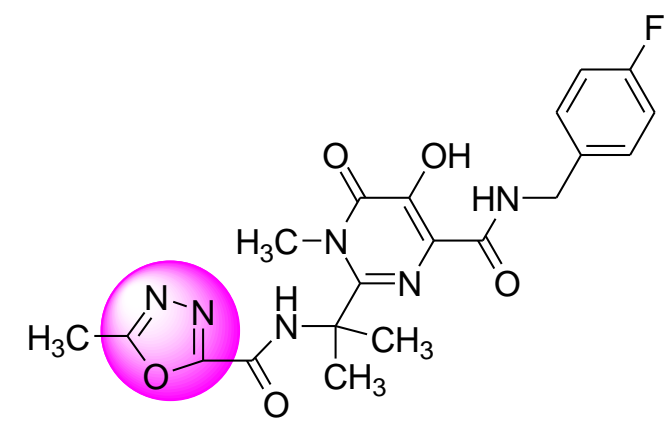

Raltegravir (Antiretroviral)<smiles>COc1cc2nc(N3CCN(C(=O)c4nnc(SCCl)s4)CC3)nc(N)c2cc1OC</smiles>

Tiodazosin

(Antihypertensive drug)

Figure 2. 1,3,4-oxadiazole based drugs in the market.

\section{Anti-cancer activity of 1,3,4-oxadiazole derivatives}

Quinoline based 1,3,4-oxadiazole, 3-(5-benzyl-1,3,4-oxadiazol-2-yl)quinolin-2(1H)one (1) and 3-[5-(2-phenoxymethyl-benzoimidazol-1-ylmethyl)-[1,3,4]oxadiazol-2-yl]-2-ptolyloxy-quinoline (2) displayed $\mathrm{GI}_{50}$ values in the range $1.41-15.8 \mu \mathrm{M}$ and $0.40-14.9 \mu \mathrm{M}$, respectively against a panel of NCI 60 cancer cell lines [28] (Scheme 1).<smiles>O=c1[nH]c2ccccc2cc1-c1nnc(Cc2ccccc2)o1</smiles>

1

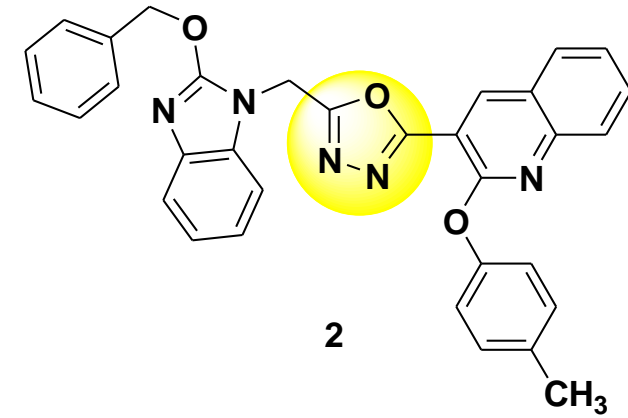

Scheme 1. Quinoline based 1,3,4-oxadiazole.

Du et al. reported 1,3,4-oxadiazole-thioether derivatives as anti-cancer agents targeting thymidylate synthase enzyme. Compound (3) was the best compound with $\mathrm{IC}_{50} 0.7 \mu \mathrm{M}, 18.3$ $\mu \mathrm{M}$, and $30.0 \mu \mathrm{M}$ against HepG2, MCF-7, and SGC-7901 cancer cells, respectively [29] (Scheme 2).

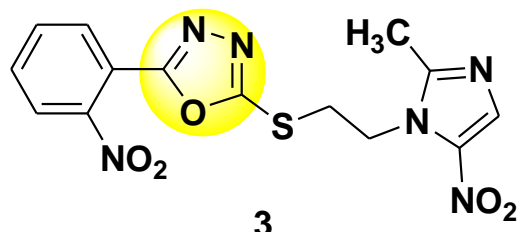

Scheme 2. 1,3,4-oxadiazole-thioether derivative. 
Adimule and his coworkers reported 2- (4'-fluorobiphenyl-3-yl)-5-(5-phenylthiophen2-yl)-1,3,4-oxadiazole (4) as potent cytotoxic agent with $\mathrm{IC}_{50} 5.3 \mu \mathrm{M}$, comparable to 5fluorouracil ( $\left.\mathrm{IC}_{50} 8.6 \mu \mathrm{M}\right)$ against Caco-2cell line [30] (Scheme 3).

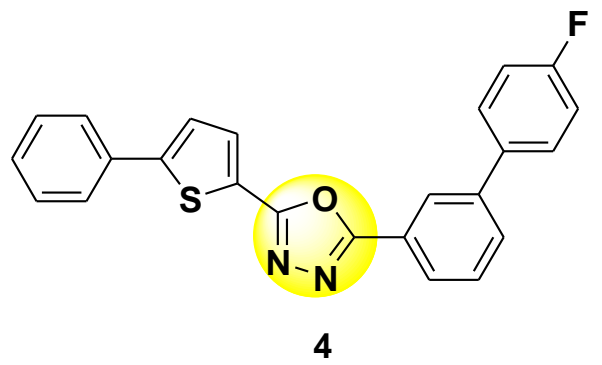

Scheme 3. Thiophene based 1,3,4-oxadiazole derivative.

Oxadiazole-based propanamide derivatives, (R)-2-amino- $\mathrm{N}-((5-$-phenyl-1,3,4oxadiazol-2-yl)methyl) propanamide (5) synthesized by Pidugu et al. has shown significant anti-cancer activity through the inhibition of Histone deacetylase (HDAC8) enzyme [31] (Scheme 4).

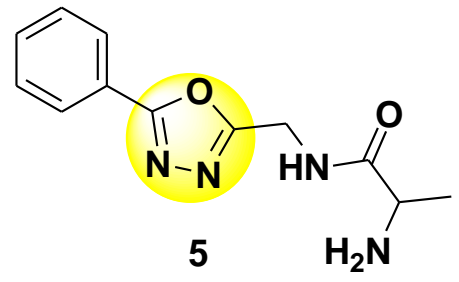

Scheme 4. Propanamide based 1,3,4-oxadiazole derivative.

Mohan et al. discovered 2-(3-chlorobenzo[b]thiophen-2-yl)-5-(3-methoxyphenyl)1,3,4-oxadiazole (6) as a potent anti-cancer agent by studying apoptosis (annexin V-propidium

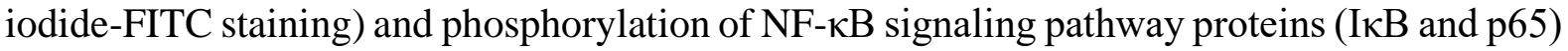
in hepatocellular carcinoma cells (HCC) [32] (Scheme 5).

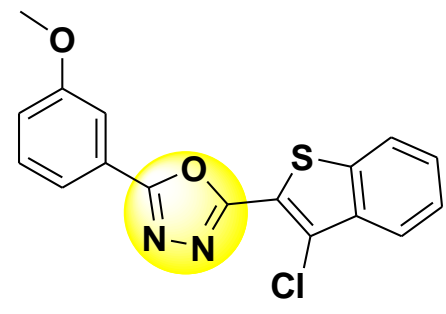

6

Scheme 5. Benzothiophene-based 1,3,4-oxadiazole derivative.

Rahman synthesized 1,3,4-oxadiazole incorporated diphenylamine derivatives and screened for anti-cancer activity. These compounds (7-9) have revealed significant cytotoxicity against $\mathrm{HT} 29$ cell line with $\mathrm{IC}_{50}$ in the range 1.3-2.0 Mm [33] (Scheme 6).

Recently, 5-bromo-1-((4-chlorophenyl)((5-(4-hydroxyphenyl)-1,3,4-oxadiazol-2yl)amino)methyl) indoline-2,3-dione (10) reported by Bhat et al. exhibited remarkable cytotoxicity with $\mathrm{IC}_{50} 0.78 \mu \mathrm{M}$ and $0.26 \mu \mathrm{M}$ against HT-29 and HepG2, respectively by inhibiting EGFR and CDK2 kinases [34] (Scheme 7). 

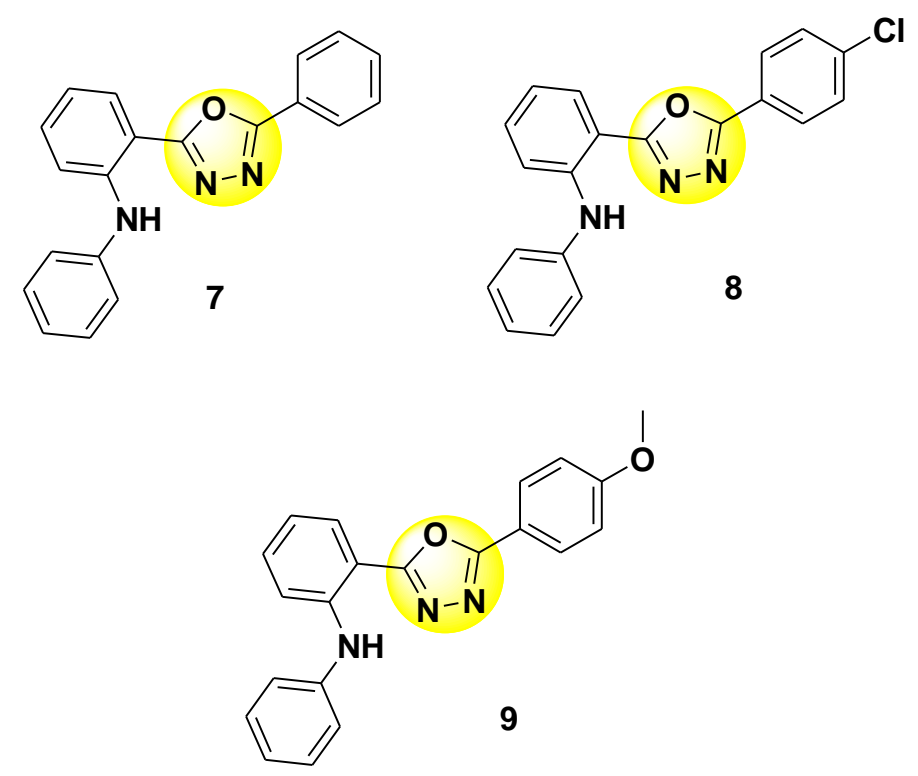

Scheme 6. Diphenylamine incorporated 1,3,4-oxadiazole derivative.<smiles>O=C1C(=O)N(C(Nc2nnc(-c3ccc(O)cc3)o2)c2ccc(Cl)cc2)c2ccc(Br)cc21</smiles>

10

Scheme 7. Indolinedione incorporated 1,3,4-oxadiazole derivative.

Kavitha and coworkers reported 1-[3-(5-Cyclohexyl-[1,3,4]oxadiazol-2-yl)-phenyl]-3-p-tolyl-urea (11) as a promising anticancer agent [35] (Scheme 8).

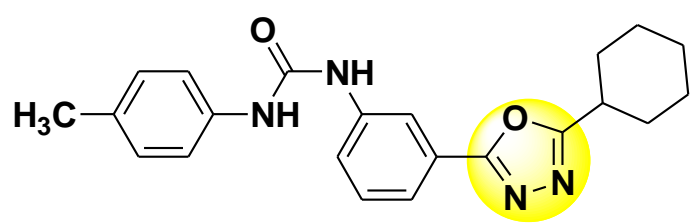

\section{1}

Scheme 8. Urea based 1,3,4-oxadiazole derivative.

Compound

$N$-(furan-2-ylmethyl)-3-[5-(furan-2-yl)-1,3,4-oxadiazol-2-yl]-6(trifluoromethyl)pyridin-2-amine (12) revealed promising anticancer activity against DU145 and HepG2 cell lines in comparison to 5-fluorouracil [36] (Scheme 9).<smiles>FC(F)(F)c1ccc(-c2nnc(-c3ccco3)o2)c(CCc2ccco2)c1</smiles>

12

Scheme 9. Furan based 1,3,4-oxadiazole derivative. 
1,3,4-oxadiazole and thiazolidine-2,4-dione based derivatives, (E)-3-\{[5-(4florophenyl)-1,3,4-oxadiazol-2-yl]methyl \}-5-(3,4,5-trimethoxybenzylidene) thiazolidine-2,4diones (13) exhibited promising cytotoxicity with $\mathrm{IC}_{50} 0.81$ to $11.9 \mu \mathrm{M}$ against $\mathrm{A} 549, \mathrm{~A} 375$, MCF-7, and HT-29 cancer cell lines[37] (Scheme 10).

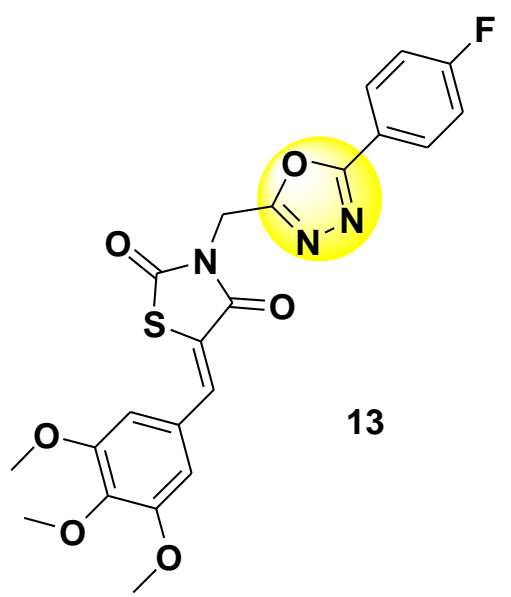

Scheme 10. Thiazolidinedione incorporated 1,3,4-oxadiazole derivative.

Zohor et al reported 1,3,4-oxadiazole-thiazolidinedione hybrids, 5-(4methoxybenzylidene)-3-((5-(2-chlorophenyl)-1,3,4-oxadiazol-2-1)methyl) thiazolidinedione (14) and 5-(4-methoxybenzylidene)-3-((5-(4-bromophenyl)-1,3,4-oxadiazol-2yl)methyl)thiazolidinedione (15) which displayed cytotoxicity with $\mathrm{IC}_{50} 7.74$ and 7.87, respectively against MCF-7 cell line through the inhibition of thymidylate synthase enzyme [38] (Scheme 11).

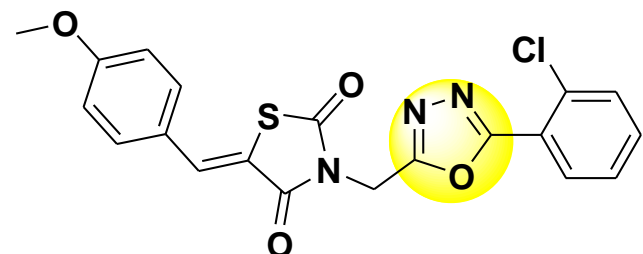

14

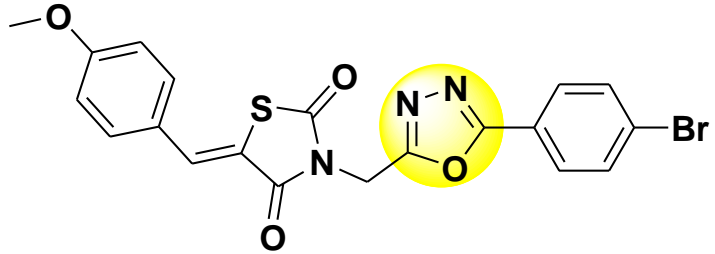

15

Scheme 11. Thiazolidinedione incorporated 1,3,4-oxadiazole derivative.

Quinoline incorporated 1,3,4-oxadiazole, 3-((2-Fluorophenyl)amino)methyl)-5(quinolin-2-yl)-1,3,4-oxadiazole-2(3H)-thione; (16) and 3-(((4-Chlorophenyl)amino)methyl)5-(quinolin-2-yl)-1,3,4-oxadiazole-2(3H)-thione (17) reported by Sun et al exhibited anticancer activity by inhibiting telomerase enzyme [39] (Scheme 12).

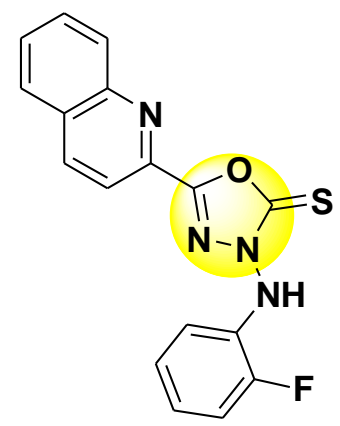

16<smiles>S=c1oc(-c2ccc3ccccc3n2)nn1Nc1ccc(Cl)cc1</smiles>

17

Scheme 12. Quinoline incorporated 1,3,4-oxadiazole derivative 
Madhavilatha et al. reported 1,3,4-oxadiazole-linked 1,2,3-triazole derivative, 3-((1Benzyl-1H-1,2,3-triazol-4-yl)methyl)-5-phenyl-1,3,4-oxadiazol-2(3H)-one (18) as promising anticancer agent against HeLa, MDA-MB-231, DU-145 and HEPG2 cancer cell lines. Compound 18 arrests cell cycle at G2/M stage and acts as a tubulin polymerization inhibitor [40] (Scheme 13).

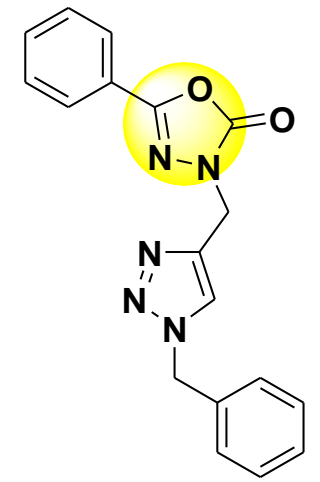

Scheme 13. 1,2,3-triazole linked 1,3,4-oxadiazole derivative.

2-((5-(4-Nitrophenyl)-1,3,4-oxadiazol-2-yl)thiomethyl)-1-benzylbenzimidazole displayed moderate cytotoxicity ( $\left.\mathrm{IC}_{50} 17.5 \mu \mathrm{M}\right)$ [41] (Scheme 14).

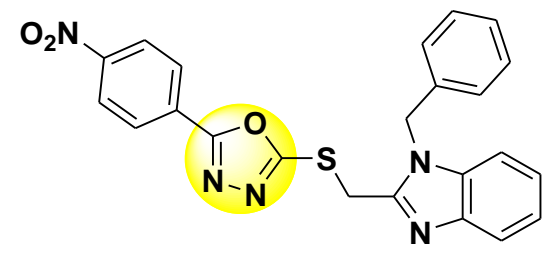

19

Scheme 14. Benzimidazole based 1,3,4-oxadiazole derivative.

Ahsan and Shastri synthesized $N$-\{[5-(4-chlorophenyl)-1,3,4-oxadiazol-2yl]methyl $\}$ pyridin-2-amine (20) and $N$-\{[5-(3,4-dimethoxyphenyl)-1,3,4-oxadiazol-2yl]methyl \}pyridin-2-amine (21) which revealed mean growth percent inhibition (GP) of 96.37 , and 95.12, respectively against NCI 60 cancer cell lines at $10 \mu \mathrm{M}$ concentration [42] (Scheme 15).

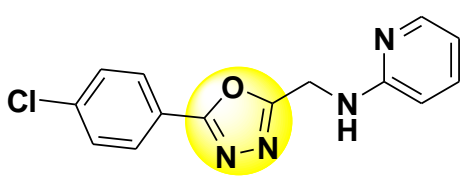

20<smiles>COc1ccc(-c2nnc(CNc3ccccn3)o2)cc1</smiles>

21

Scheme 15. Pyridine based 1,3,4-oxadiazole derivative.

Vinayak et al reported \{5-[6-(4-Fluorophenyl)-pyridin-3-yl]-[1,3,4] oxadiazol-2-ylmethyl $\}$-phenyl-amine (22) as a potent cytotoxic agent with $\mathrm{IC}_{50} 2.3 \mu \mathrm{M}$ against Caco-2 cell lines [43] (Scheme 16).

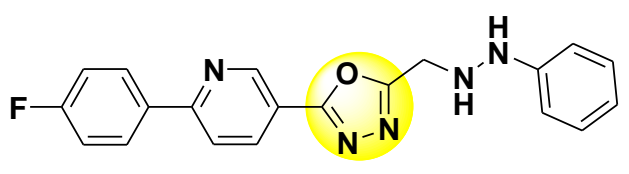

22

Scheme 16. Pyridine based 1,3,4-oxadiazole derivative. 
Novel 1,3,4-oxadiazole linked benzoxazole analogues, N1-(4-Methoxyphenyl)-2-\{5[4-(1,3-benzoxazol-2-yl)phenyl]-1,3,4-oxadiazol-2-ylsulfanyl \}acetamide (23) has shown potent antiproliferative effect with $\mathrm{IC}_{50}$ of 0.018 against HT-29cancer cell line [44] (Scheme 17).

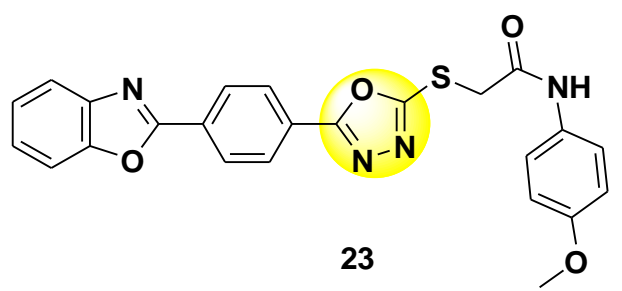

Scheme 17. Benzoxazole based 1,3,4-oxadiazole derivative.

Khalil and colleagues reported N'-[(Z/E)-(3-Indolyl)methylidene]-2-\{[5-(pyridin-4yl)-1,3,4- oxadiazol-2-yl]sulfanyl \}acetohydrazide (24) which has shown significant anticancer effect $\left(\mathrm{IC}_{50}: 0.010 \mu \mathrm{M}\right)$ through the inhibition of EGFR [45] (Scheme 18).<smiles>O=C(CSc1nnc(-c2ccncc2)o1)N/N=C1\CNc2ccccc21</smiles>

Scheme 18. Indole based 1,3,4-oxadiazole derivative.

Quinazoline based 1,3,4-oxadiazole, 3-[5-(4-fluorophenyl)-1,3,4-oxadiazol-2-yl]-2phenylquinazolin-4(3H)-one (25) exhibited cytoxicity with $\mathrm{IC}_{50} 17.7 \mu \mathrm{M}$ against $\mathrm{K} 562$ cell lines, compared to doxorubicin [46] (Scheme 19).

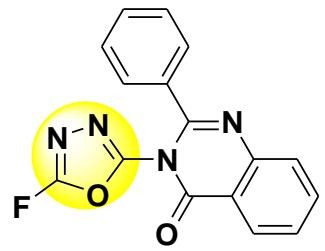

25

Scheme 19. Quinazoline based 1,3,4-oxadiazole derivative.

2-(3,4,5-trimethoxyphenyl)-5-(4-(5-(3,4,5- trimethoxyphenyl)-1,2,4-oxadiazol-3yl)phenyl)-1,3,4-oxadiazole (26) containing 1,3,4-oxadiazole and 1,2,4-oxadiazole moieties displayed potent anticancer effect with $\mathrm{IC}_{50} 0.34$ to $2.45 \mu \mathrm{M}$ against all the tested MCF-7, A549 and MDA-MB-231 cancer cells by inhibiting EGFR (epidermal growth factor receptor) [47] (Scheme 20).

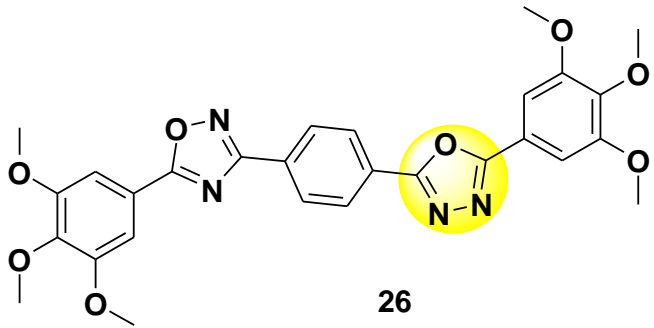

Scheme 20. 1,2,4-oxadiazole linked 1,3,4-oxadiazole derivative.

2-(5-(4-chlorophenyl)-1,3,4-oxadiazol-2-ylthio) N-(4-oxo-2-propylquinazolin)-3-(4H) acatamide (27) synthesized by Hassanzadeh et al. displayed promising anti-cancer effect with $\mathrm{IC}_{50}$ of $7.52 \mu \mathrm{M}$ against the HeLa cell line [48] (Scheme 21). 


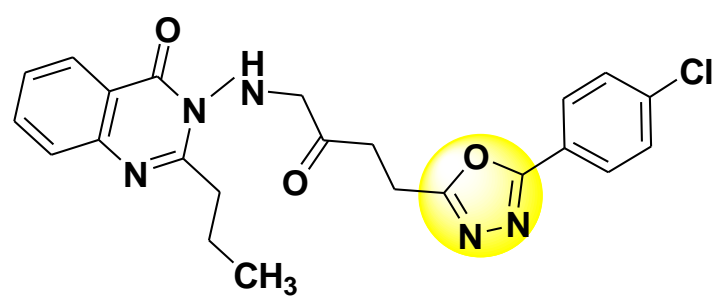

27

Scheme 21. Quinazoline based 1,3,4-oxadiazole derivative.

1,3,4-oxadiazole tethered benzimidazoles, 1-(1Hbenzo[d]imidazol-2-yl)-3-(5-(2,4dichlorophenyl)-1,3,4- oxadiazol-2-yl)propan-1-one (28), was found to be a hit compound against NCI 60 cancer cell line [49] (Scheme 22).

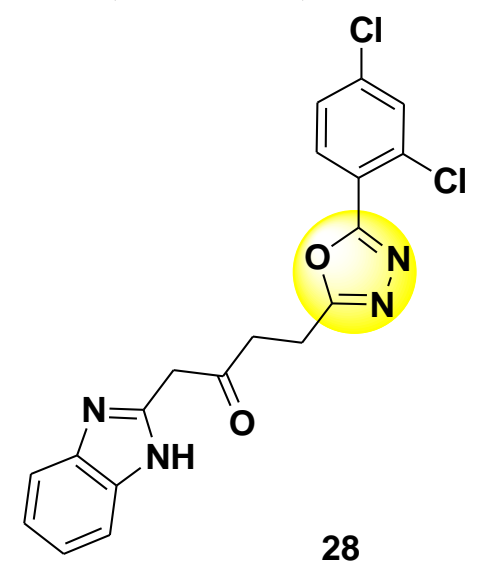

Scheme 22. Benzimidazole based 1,3,4-oxadiazole derivative.

Pyrazole-based 1,3,4-oxadiazole, N-methyl- 4-(trifluoromethyl) phenyl pyrazole (29) exerted an antiproliferative effect with $\mathrm{IC}_{50}$ of $15.54 \mu \mathrm{M}$, compared to doxorubicin [50] (Scheme 23).

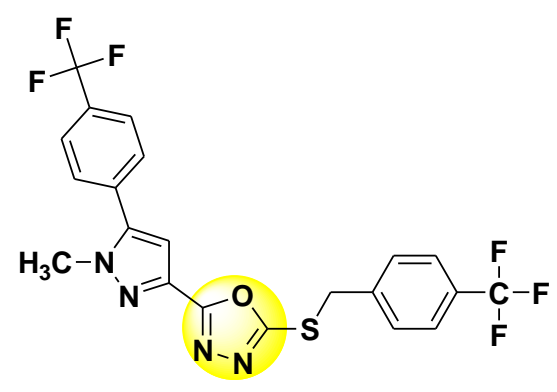

29

Scheme 23. Pyrazole based 1,3,4-oxadiazole derivative.

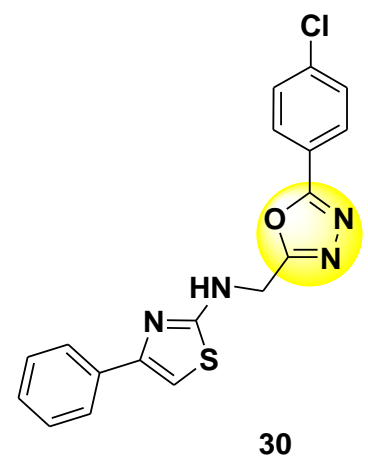

Scheme 24. Thiazole based 1,3,4-oxadiazole derivative. 
Jisha et al. synthesized $N$-\{[5-(4-chlorophenyl)-1,3,4-oxadiazol-2-yl] methyl $\}-4-$ phenyl-1,3-thiazol-2-amine (30) which revealed promising cytotoxicity against both DLA and MCF-7 with $\mathrm{LD}_{50} 136 \mu \mathrm{g} / \mathrm{ml}$ and $132 \mu \mathrm{g} / \mathrm{ml}$, respectively [51] (Scheme 24).

Kapoor and Dhiman reported 2-(4-dimethoxyphenyl)-1-\{2-[5-(2-methoxyphenyl1,3,4-oxadiazole-2-yl]phenyl $\}-1 H$ benzimidazole (31) with potent cytoxicity against MCF-7 cells [52] (Scheme 25).

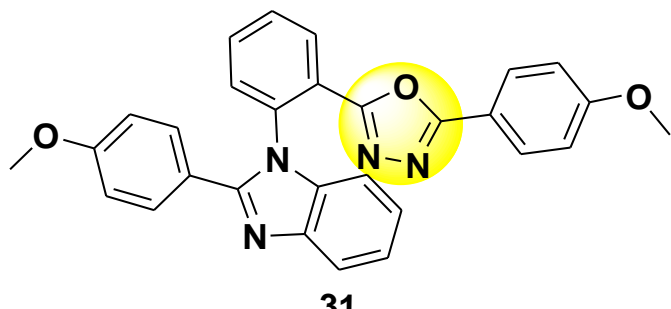

31

Scheme 25. Benzimidazole based 1,3,4-oxadiazole derivative.

Abdo and Kamel et al. reported a series of 5-(pyridin-4-yl)- $N$-substituted-1,3,4oxadiazol-2-amines (32-34) which exhibited cytotoxicity in the range of 0.725-3.274 $\mu \mathrm{m}$ against all six human cancer cell lines, NUGC, DLD1, HA22T, HEPG2, HONE1 MCF cells [53] (Scheme 26).

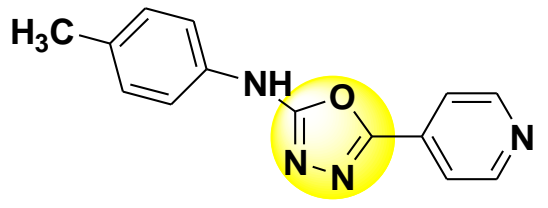

32

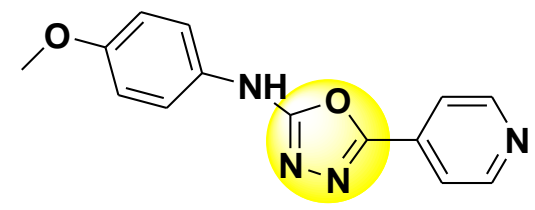

33

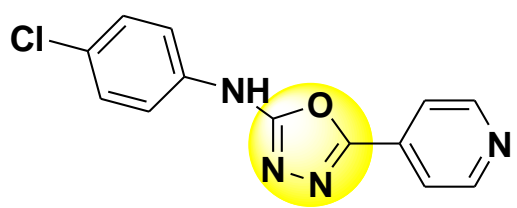

34

Scheme 26. Pyridine based 1,3,4-oxadiazole derivative.

Ghag and Kamath reported (E)-1-phenyl-4-(5-styryl-1,3,4-oxadiazol-2-yl)-1Hpyrazol5-amine (35) with excellent activity than standard drug cisplatin on breast cell lines DU145 [54] (Scheme 27).<smiles>Nc1c(-c2nnc(/C=C/c3ccccc3)o2)cnn1-c1ccccc1</smiles>

35

Scheme 27. Pyrazole based 1,3,4-oxadiazole derivative.

Benzenesulfonamide linked 1,3,4-oxadiazole, (E)-2-benzylthio-4-chloro-5- (5-styryl1,3,4-oxadiazol-2-yl)benzenesulfonamide (36) reported by Sławinskiet al. has displayed promising anti-cancer activity against HCT-116, MCF-7 and HeLa cancer cell lines with IC 50 : 11-29 $\mu \mathrm{M}[55]$ (Scheme 28). 


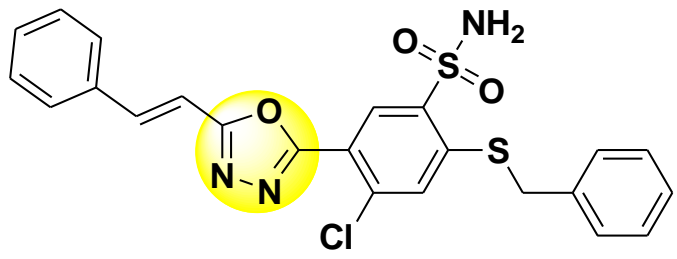

36

Scheme 28. Benzenesulfonamide based 1,3,4-oxadiazole derivative.

Benzothiazole clubbed 1,3,4-oxadiazole, 2-(5-((benzo[d]thiazol-2-yloxy)-methyl)1,3,4-oxadiazol-2-yl)phenol (37) reported by Alghamdi et al showed cytotoxicity effect with IC $_{50} 1.8 \mu \mathrm{M} / \mathrm{mL}$, which was comparable to the standard drug, Doxirubicin (IC $501.2 \mu \mathrm{M} / \mathrm{mL}$ ) [56] (Scheme 29).

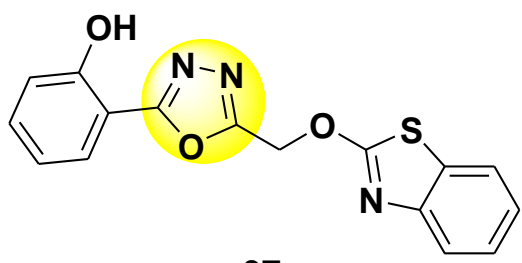

37

Scheme 29. Benzothiazole linked 1,3,4-oxadiazole derivative.

Alam et al. reported 4-((5-phenyl-1,3,4-oxadiazol-2-ylthio)methyl)-1-phenyl-1H1,2,3-triazole (38) as the potent cytotoxic agent which showed significant inhibitory effects on the viability of MCF-7 and HCT-116 cells with $\mathrm{IC}_{50} 5.80$ and $14.80 \mu \mathrm{M}$, respectively [57] (Scheme 30).

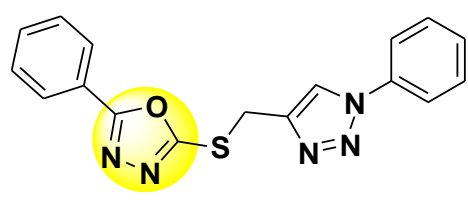

38

Scheme 30. 1,2,3-triazole linked 1,3,4-oxadiazole derivative.

Ibuprofen-N-acyl-1,3,4-oxadiazole derivatives (39) reported by Alderawi et al. has shown significant cytotoxicity with $85.1 \%$ inhibition against MCF-7 cells [58] (Scheme 31).

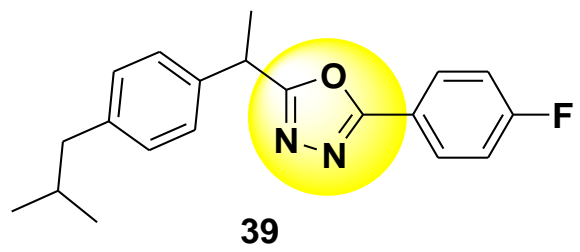

Scheme 31. Ibuprofen based 1,3,4-oxadiazole derivative.

Han et al. reported 2-phenyl-4H-chromone derivatives containing amide and 1,3,4oxadiazole moiety (40) as potential telomerase inhibitors with $\mathrm{IC}_{50} 0.44 \mu \mathrm{M}$, compared to the standard drug, Staurosporine ( $\left.\mathrm{IC}_{50} 6.41 \mu \mathrm{M}\right)$. Compound 40 also arrests MGC-803 cell cycle at G2/M phase and induce apoptosis [59] (Scheme 32). 


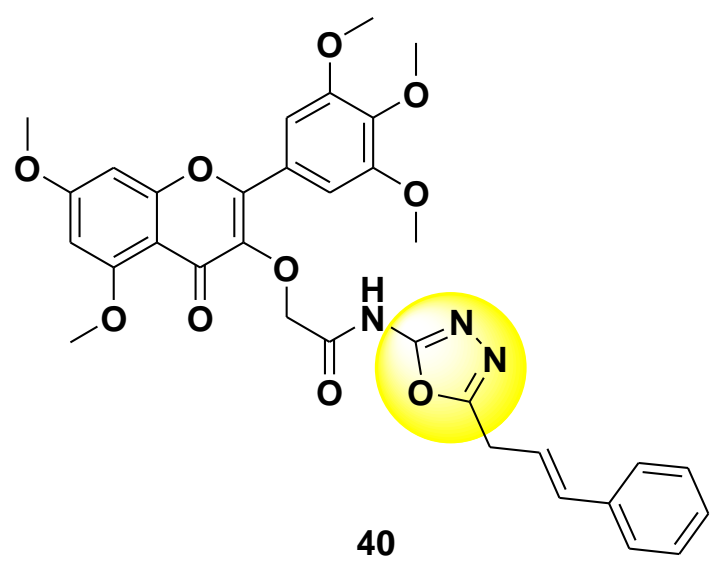

Scheme 32. Chromone based 1,3,4-oxadiazole derivative.

Indazole tethered oxadiazoles derivatives, 2-(4-methoxyphenyl)-5-(1-methyl- 1Hindazol-3-yl)-1,3,4-oxadiazole (41) was found to exhibit anti-cancer activity by inhibiting the catalytical activity of SIRT2 with $\mathrm{IC}_{50} 19.5 \mu \mathrm{M}$ against HepG2 cancer cells [60] (Scheme $33)$.

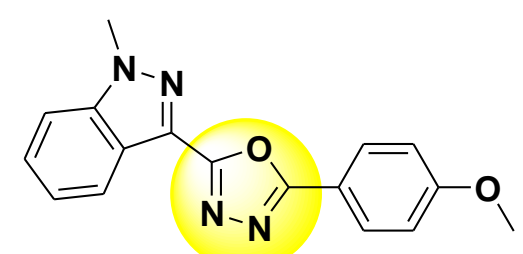

41

Scheme 33. Indazole based 1,3,4-oxadiazole derivative.

Malojirao discovered 2-(3-(6-chloro-5-methylpyridin-3-yl)phenyl)-5-(1-methyl-1Hindazol-3-yl)-1,3,4-oxadiazole (CHK9) (42) as a potent cytotoxic agent with IC 50 values ranging between 4.8-5.1 $\mu \mathrm{M}$ against A549, MCf-7, A375, HepG2, Huh-7.ACHN, A498, and LLC cells [61] (Scheme 34).

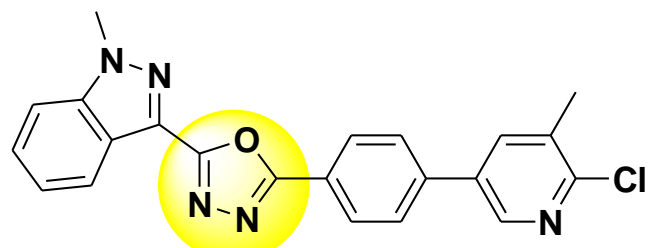

42

Scheme 34. Indazole based 1,3,4-oxadiazole derivative.

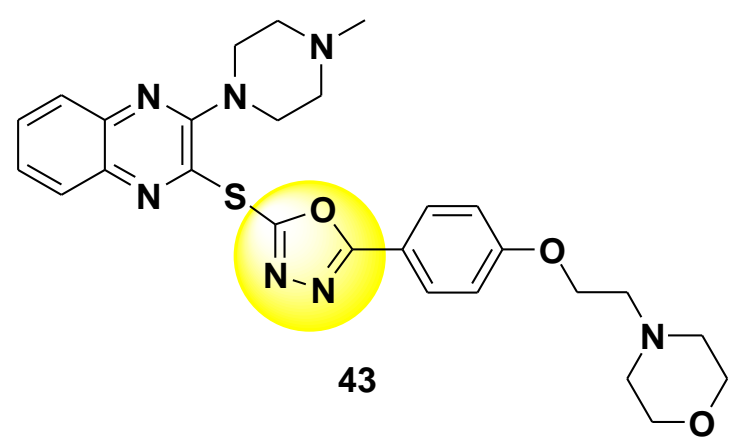

Scheme 35. Benzopyran based 1,3,4-oxadiazole derivative. 
New benzopyran 1,3,4-oxadiazole, derivatives designed by Kumar et al., displayed potent anti-cancer activity on the MCF-7 cell line [62]. Quinoxaline-1,3,4-oxadiazole hybrid (43) displayed noteworthy cytotoxicity with $90 \%$ cell viability against cancer cells by inducing apoptosis through downregulation of Bcl-2 expression [63] (Scheme 35).

Compound 1-(5-(Pyridin-4-yl)-2-p-tolyl-1,3,4-oxadiazol-3(2H)-yl)ethanone exhibited equipotent cytotoxic activity with an $\mathrm{IC}_{50}$ value of $8.04 \mu \mathrm{M}$ when compared with that of standard drug doxorubicin (IC50=8.02 $\mu \mathrm{M})[64]$ (Scheme 36).

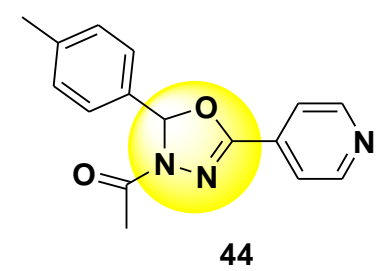

Scheme 36. Pyridine based 1,3,4-oxadiazole derivative.

Novel theophylline-based 1,3,4-oxadiazole derivatives, (45) and (46) reported by Gopinatha et al.showed 6.25-8.6 fold better cytotoxicity than SCR7 in leukemic cell lines, CEM [65] (Scheme 37).
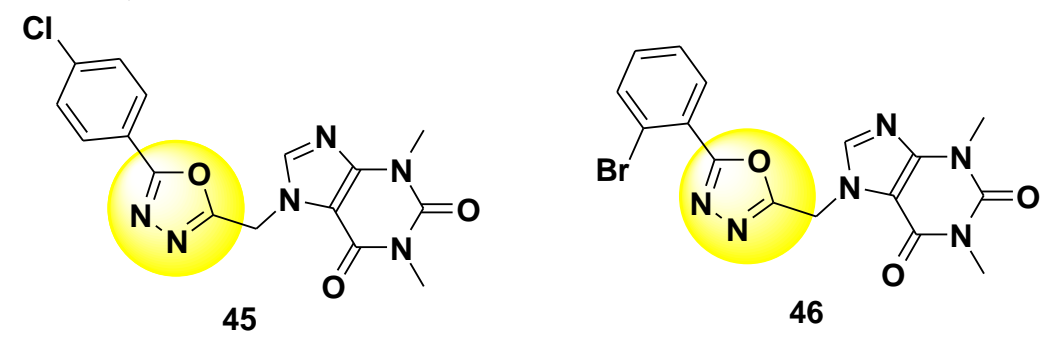

Scheme 37. Theophylline based 1,3,4-oxadiazole derivative.

Novel Ursane 1,3,4-oxadiazole derivatives, $\quad \beta$-(4-Oxo-4-((1-((5-phenyl-1,3,4oxadiazol-2-yl)methyl)-1H-1,2,3-triazol-4-yl)-methoxy)-butanoyloxy)-urs-12-en-28-oic acid (47) was found to be toxic with $\mathrm{IC}_{50} 40.26 \mu \mathrm{M}$ towards A549 lung cancer cell line [66] (Scheme 38).

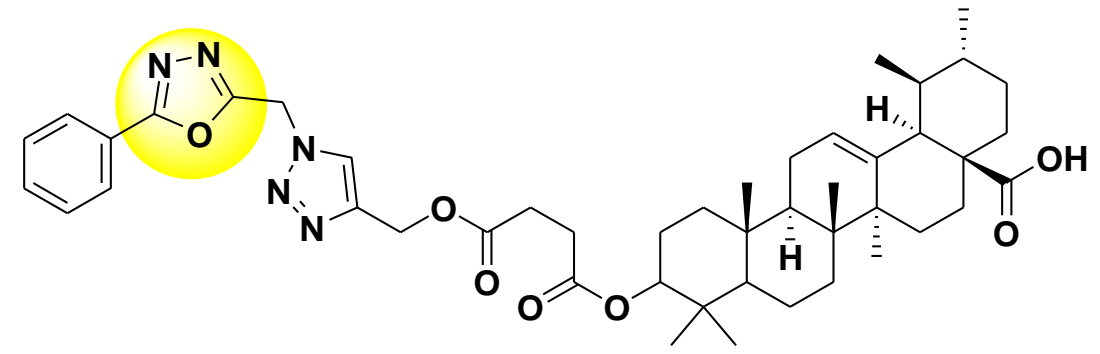

47

Scheme 38. Ursane based 1,3,4-oxadiazole derivative.

Resveratrol fused 1,3,4-oxadiaozle derivatives, (48) and (49) revealed better anticancer activity with $\mathrm{IC}_{50}$ in the range $0.11 \mu \mathrm{M}-1.56 \mu \mathrm{M}$ and $\mathrm{IC}_{50} 0.45 \mu \mathrm{M}-1.98 \mu \mathrm{M}$, respectively standard drug Adriamycin showed $\mathrm{IC}_{50}$ in the range $2.10 \mu \mathrm{M}-3.41 \mu \mathrm{M}$ against MCF-7, A549 and MDA-MB-231 cells [67] (Scheme 39). 


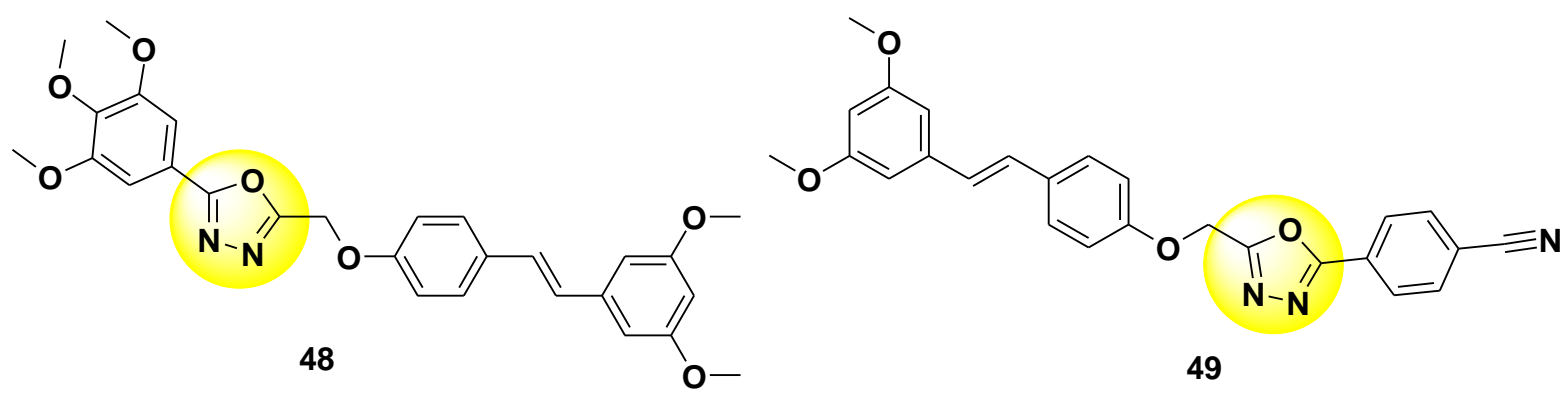

Scheme 39. Resveratrol based 1,3,4-oxadiazole derivative.

Polkam et al. reporetd 2-(2,5-dimethoxyphenyl)-5-propylthio-1,3,4-oxadiazole (50) and 2-(2,5-Dimethoxyphenyl)-5-butylthio-1,3,4-oxadiazole (51) as promising anticancer agents with $\mathrm{IC}_{50} 16.66 \mu \mathrm{M}$ and $8.26 \mu \mathrm{M}$, respectively against prostate cancer cell line, DU145 [68] (Scheme 40).

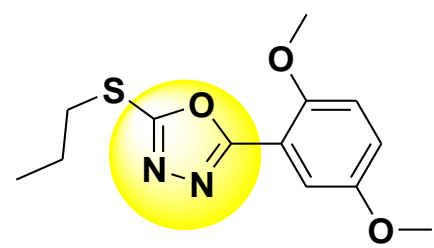

50

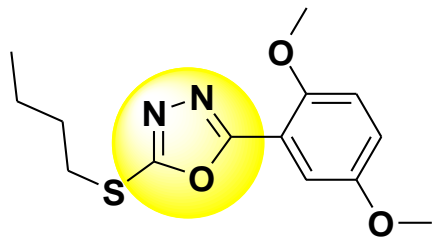

51

Scheme 40. Thioether based 1,3,4-oxadiazole derivative.

Abdelrehim reported 2-[5-(1-Methyl-1H-pyrrol-3-yl)-1-phenyl-1H-pyrazol-3-yl]-5methylsulfanyl-[1,3,4]oxadiazole (52) which showed mild cytotoxicity on colon cancer cell, HCT-116 [69] (Scheme 41).

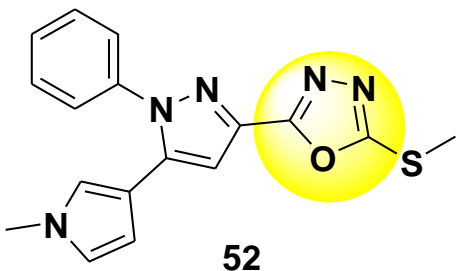

Scheme 41. Pyrazole based 1,3,4-oxadiazole derivative.

Novel pyrimidine-oxazole based 1,3,4-xadiaozle hybrids (53) demonstrated significant anti-cancer activity with $\mathrm{IC}_{50}$ values in the range $0.011 \mu \mathrm{M}$ to $19.4 \mu \mathrm{M}$ in comparison to reference drug, etoposide ( $\mathrm{IC}_{50} 0.13 \mu \mathrm{M}$ to $3.08 \mu \mathrm{M}$ ) towards MCF-7, A549, Colo-205 and A2780 cell lines [70] (Scheme 42).<smiles>O=[N+]([O-])c1cc(-c2nnc(-c3cn4c(n3)oc3ccc(-c5cncnc5)cc34)o2)cc([N+](=O)[O-])c1Br</smiles>

53

Scheme 42. Benzoxazole based 1,3,4-oxadiazole derivative.

Erensoy and coworkers reported 2-[5-\{[2-methyl-5-(propan-2-yl)phenoxy]methyl $\}$ 1,3,4-oxadiazol-2-yl)sulphanyl]-1-(phenyl)ethan-1-one (54) which showed inhibitory activity against mPGES-1 with an IC 50 of $4.95 \mu \mathrm{M}$ [71] (Scheme 43). 


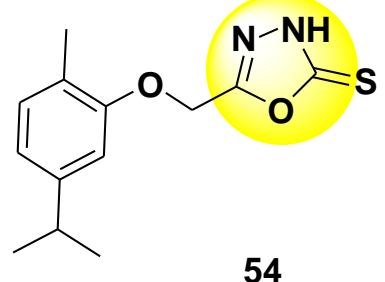

Scheme 43. Cravacrol- 1,3,4-oxadiazole hybrid.

Novel benzimidazole-1,3,4-oxadiazole hybrids (55) and (56) exhibited the potent antiproliferative activity than Hoechst 33342 and doxorubicin against HeLa cell line, with $\mathrm{IC}_{50}$ of $0.224 \mu \mathrm{M}$ and $0.205 \mu \mathrm{M}$, respectively by inhibiting topoisomerase I enzyme [72] (Scheme 44).<smiles>CCOc1ccc(-c2nc3ccc(-c4nnc(SCC(=O)N5CCN(CC)CC5)o4)cc3[nH]2)cc1</smiles>

Scheme 44. Benzimidazole fused 1,3,4-oxadiazole hybrid.

\section{Conclusions}

The article reports the different oxadiazole derivatives, which have shown significant anti-cancer potential. 1,3,4-oxadiazole is a privileged heterocycle with remarkable potential to discover new leads for cancer treatment. Furthermore, it has the potential to inhibit different enzymes which are involved in cell proliferation. The present review will give a platform to the medicinal chemists to develop new and potent 1,3,4-oxadiazole derivatives with better efficacy and reduced side effects.

\section{Funding}

This research received no external funding.

\section{Acknowledgments}

The author thanks the Chemistry department, Albaha University, for providing the necessary facilities to complete this review article.

\section{Conflicts of Interest}

The authors declare no conflict of interest.

\section{References}

1. Manikandan, A.; Antony, S.A.; Sridhar, R.; Ramakrishna, S.; Bououdina, M. A Simple Combustion Synthesis and Optical Studies of Magnetic Zn1-x Ni x Fe2O4 Nanostructures for Photoelectrochemical Applications.Journal of Nanoscience and Nanotechnology2015, 15, 49484960,https://doi.org/10.1166/jnn.2015.9814.

2. Chitra, K.; Manikandan, A.; Moortheswaran, S.; Reena, K.; Antony, S.A. Zingiber officinale extracted green synthesis of copper nanoparticles: structural, morphological and antibacterial studies. Advance Science Engineering Medicine2015, 7, 710-716,https://doi.org/10.1166/asem.2015.1752. 
3. Subramanian, A.P.; Jaganathan, S. K.; Manikandan, A.; Pandiaraj, K.N.; Nageswaran, G.; Supriyanto E. Recent trends in nano-based drug delivery systems for efficient delivery of phytochemicals in chemotherapy. RSC Advances2016, 6, 48294-48314,https://doi.org/10.1039/C6RA07802H.

4. Vellayappan, M.V.; Venugopal, J.R.; Ramakrishna, S.; Ray, S.; Ismail, A.F.; Mandal, M.; Manikandan, A.; Seal, S.; Jaganathan, S.K. Electrospinning applications from diagnosis to treatment of diabetes. RSC Advances 2016, 6, 83638-83655,https://doi.org/10.1039/C6RA15252J.

5. Vellayappan, M.; Jaganathan, S.; Manikandan, A. Nanomaterials as a game changer in the management and treatment of diabetic foot ulcers. RSC Advances 2016, 6, 114859114878,https://doi.org/10.1039/C6RA24590K.

6. Rehman, A.; Siddiqa, A.; Abbasi, M.A.; Rasool, S.; Siddiqui, S. Z.; Ahmad, I.; Afzal, S. Synthesis of some new 5-substituted-2-((6-chloro-3, 4-methylenedioxyphenyl) methylthio)-1, 3, 4-Oxadiazole derivatives as suitable antibacterial inhibitors. Bulletin of Faculty of Pharmacy, Cairo University2015, 53, 3743,https://doi.org/10.1016/j.bfopcu.2014.10.001.

7. Song, X.; Li, P.; Li, M.; Yang, A.; Yu, L.; Luo, L.; Hu, D.; Song, B. Synthesis and investigation of the antibacterial activity and action mechanism of 1,3,4-oxadiazole thioether derivatives. Pesticide Biochemistry and Physiology2018, 147, 11-19,https://doi.org/10.1016/j.pestbp.2017.10.011.

8. Wani. M.Y.; Ahmad, A.; Shiekh, R.A.; Al-Ghamdi, K.J.; Sobral, A.J. Imidazole clubbed 1, 3, 4-oxadiazole derivatives as potential antifungal agents. Bioorganic Medicinal Chemistry2015,23, 41724180,https://doi.org/10.1016/j.bmc.2015.06.053.

9. Othman, A.A.; Kihel, M.; Amara, S. 1, 3, 4-Oxadiazole, 1, 3, 4-thiadiazole and 1, 2, 4-triazole derivatives as potential antibacterial agents. Arabian Journal of Chemistry2014, 12, 16601675,https://doi.org/10.1016/j.arabjc.2014.09.003.

10. Zhou, L.; Wang, P.Y.; Zhou, J.D.; Shao, W.B.; Fang, H.S.; Wu, Z.; Yang, S. Antimicrobial activities of pyridinium-tailored pyrazoles bearing 1, 3, 4-oxadiazole scaffolds. Journal of Saudi Chemical Soceity2017, 21, 852-860,https://doi.org/10.1016/j.jscs.2017.04.005.

11. Guo, Z.H.; Yin, Y.; Wang, C.; Wang, P.F.; Zhang, X.T.; Wang, Z.C.; Zhu, H.L. Design, synthesis and molecular docking of salicylic acid derivatives containing metronidazole as a new class of antimicrobial agents. Bioorganic Medicinal Chemistry2015, 23, 6148-6156,https://doi.org/10.1016/j.bmc.2015.07.075.

12. Mullican, M.D.; Wilson, M.W.; Connor, D.T.; Kostlan, C.R.; Schrier, D.J.; Dyer, R.D. Design of 5-(3, 5-ditert-butyl-4-hydroxyphenyl)-1, 3, 4-thiadiazoles,-1, 3, 4-oxadiazoles, and-1, 2, 4-triazoles as orally active, nonulcerogenic anti-inflammatory agents. Journal of Medicinal Chemistry1993, 36, 10901099,https://doi.org/10.1021/jm00060a017.

13. Youssef, A.M.; White, M.S.; Villanueva, E.B.; El-Ashmawy, I.M.; Klegeris, A. Synthesis and biological evaluation of novel pyrazolyl-2, 4-thiazolidinediones as anti-inflammatory and neuroprotective agents. Bioorganic Medicinal Chemistry2010, 18, 2019-2028,https://doi.org/10.1016/j.bmc.2010.01.021.

14. Jayashankar, B.; Rai, K.M.L.; Baskaran, N.; Sathish, H.S. Synthesis and pharmacological evaluation of 1, 3, 4-oxadiazole bearing bis (heterocycle) derivatives as anti-inflammatory and analgesic agents. European Journal of Medicinal Chemistry2009, 44, 3898-3902,https://doi.org/10.1016/j.ejmech.2009.04.006.

15. Ragab F.A.F.; Abou-Seri, S.M.; Abdel-Aziz, S.A.; Alfayomy, A.M.; Aboelmagd, M. Design, synthesis and anti-cancer activity of new monastrol analogues bearing 1, 3, 4-oxadiazole moiety. European Journal of Medicinal Chemistry2017, 138, 140-151,https://doi.org/10.1016/j.ejmech.2017.06.026.

16. Mareddy, J.; Suresh, N.; Kumar, C.G.; Kapavarapu, R.; Jayasree, A.; Pal S. 1, 2, 3-Triazole-nimesulide hybrid: Their design, synthesis and evaluation as potential anti-cancer agents. Bioorganic Medicinal Chemistry Letters2017, 27, 518-523,https://doi.org/10.1016/j.bmcl.2016.12.030.

17. Kumar, D.; Patel, G.; Chavers, A.K.; Chang, K.H.; Shah, K. Synthesis of novel 1, 2, 4-oxadiazoles and analogues as potential anti-cancer agents. European Journal of Medicinal Chemistry2011, 46, 30853092,https://doi.org/10.1016/j.ejmech.2011.03.031.

18. Jha, K.K.; Abdul Samad; Kumar, Y.; Shaharyar, M.; Khosa, R. L.; Jain, J.; Kumar, V.; Singh, P. Design, synthesis and biological evaluation of 1, 3, 4-oxadiazole derivatives. European Journal of Medicinal Chemistry2010, 45, 4963-4967,https://doi.org/10.1016/j.ejmech.2010.08.003.

19. Musad, E.A.; Mohamed, R.; Saeed, B.A.; Vishwanath, B.S.; Rai, K.M. Synthesis and evaluation of antioxidant and antibacterial activities of new substituted bis (1, 3, 4-oxadiazoles), 3, 5-bis (substituted) pyrazoles and isoxazoles. Bioorganic Medicinal Chemistry Letters2011, 21, 35363540,https://doi.org/10.1016/j.bmcl.2011.04.142.

20. Gan, X.; Hu, D.; Chen, Z.; Wang, Y.; Song, B. Synthesis and antiviral evaluation of novel 1, 3, 4oxadiazole/thiadiazole-chalcone conjugates. Bioorganic Medicinal Chemistry Letters2017, 27, 42984301,https://doi.org/10.1016/j.bmcl.2017.08.038.

21. Wu, W.; Chen, Q.; Tai, A.; Jiang, G.; Ouyang, G. Synthesis and antiviral activity of 2-substituted methylthio5-(4-amino-2-methyl pyrimidin-5-yl)-1,3,4-oxadiazole derivatives. Bioorganic Medicinal Chemistry Letters2015, 25, 2243-2246,https://doi.org/10.1016/j.bmcl.2015.02.069.

22. Nazreen, S.; Alam, M.S.; Hamid, H.; Yar, M.S.; Shafi, S.; Dhulap, A.; Alam, P.; Pasha, M.A.; Bano, S.; Alam, M.M.; Haider, S.; Ali, Y.; Kharbanda, C.; Pillai, K.K. Design, synthesis, in silico molecular docking and biological evaluation of novel oxadiazole based thiazolidine-2, 4-diones bis-heterocycles as PPAR- $\gamma$ 
agonists. European Journal of Medicinal Chemistry2014, $\quad 87, \quad 175-$ 185,https://doi.org/10.1016/j.ejmech.2014.09.010.

23. Bhutani, R.; Pathak, D.P.; Kapoor, G.; Husain, A.; Kant, R.; Iqbal, M.A. Synthesis, molecular modelling studies and ADME prediction of benzothiazole clubbed oxadiazole-Mannich bases, and evaluation of their anti-diabetic activity through in vivo model. Bioorganic Chemistry2018, 77, 615,https://doi.org/10.1016/j.bioorg.2017.12.037.

24. Adachi, C.; Tsutsui, T.; Saito, S. Blue light-emitting organic electroluminescent devices. Applied Physisc Letters1990, 56, 799-801,http://dx.doi.org/10.1063/1.103177.

25. Paun, A.; Hadade, N.; Paraschivescu, C.; Matache, M. 1, 3, 4-Oxadiazoles as luminescent materials for organic light emitting diodes via cross-coupling reactions. Journal of Materials Chemistry C. 2016,4, 85968610,https://doi.org/10.1039/C6TC03003C.

26. Wei, H.; He, C.; Zhang, J.; Shreeve, J.M. Combination of 1, 2, 4-Oxadiazole and 1, 2, 5-Oxadiazole Moieties for the Generation of High-Performance Energetic Materials. Angewandte Chemie International Edition, England2015, 54, 9367-9371,https://doi.org/10.1002/anie.201503532.

27 Glomb, T.; Szymankiewicz, K.; Swiatek, P. Anti-Cancer Activity of Derivatives of 1,3,4-Oxadiazole. Molecules2018, 23, https://doi.org/10.3390/molecules23123361.

28. Salahuddin, M.A.; Shaharyar, M. Synthesis, characterization, and in vitro anti-cancer evaluation of novel 2,5disubstituted 1,3,4-oxadiazole analogue. Biomedical Research 2014, 14,https://doi.org/10.1155/2014/491492.

29. Du, Q.R.; Li, D.D.; Pi, Y.Z.; Li, J.R.; Sun, J.; Fang, F. Novel 1,3,4-Oxadiazole Thioether Derivatives Targeting Thymidylate Synthase as Dual Anticancer /Antimicrobial Agents. Bioorganic Medicinal Chemistry2013, 21, 2286-2297,https://doi.org/10.1016/j.bmc.2013.02.008.

30. Adimule, V.; Medapa, S.; Jagadeesha, A.H.; Kumar, L.S.; Rao, P.K. Synthesis, characterization and cytotoxic evaluation of novel derivatives of 1, 3, 4-oxadiazole containing 5-phenyl thiophene moiety. International Journal of Pharma and Biosciences2014, 9, 42-48,https://doi.org/10.9790/3008-09544248.

31. Pidugu, V.R.; Yarla, N.S.; Pedada, S.R.; Kalle, A.M.; Satya, A.K. Design and synthesis of novel HDAC8 inhibitory 2, 5-disubstituted-1, 3, 4-oxadiazoles containing glycine and alanine hybrids with anti-cancer activity. Bioorganic Medicinal Chemistry2016, 24, 5611-5617,https://doi.org/10.1016/j.bmc.2016.09.022.

32. Mohan, C.D.; Anilkumar, N.C.; Rangappa, S.; Shanmugam, M.K.; Mishra, S.; Chinnathambi, A. Novel 1,3,4Oxadiazole induces anti-cancer activity by Targeting $\mathrm{nF}-\mathrm{\kappa B}$ in hepatocellular carcinoma cells. Frontiers in Oncology2018, 8, https://doi.org/10.3389/fonc.2018.00042.

33. Rahman, D.E.A. Synthesis, quantitative structure-activity relationship and biological evaluation of 1,3,4-oxadiazole derivatives possessing diphenylamine moiety as potential anti-cancer agents. Chemical and Pharmaceutical Bulletin2013, 61, 151-159, https://doi.org/10.1248/cpb.c12-00637.

34. Bhat, S.A.; Jha, A. Design and ultrasound assisted synthesis of novel 1,3,4-oxadiazole drugs for anti-cancer activity. Chemistry Select2020, 5, 3347-3354,https://doi.org/10.1002/slct.201904412.

35. Kavitha,S.; Kannan, K.; Gnanavel,S. Synthesis, characterization and biological evaluation of novel 2,5 substituted-1,3,4 oxadiazole derivatives. Saudi Pharmaceutical Journal2017, 25, 337345,https://doi.org/10.1016/j.jsps.2016.07.004.

36. Kumar, R.N.; Poornachandra, Y.; Nagender, P.; Kumar, G.S.; Swaroop, D.K.; Kumar, C.G.; Narsaiah, B. Synthesis of novel nicotinohydrazide and (1,3,4-oxadiazol-2-yl)-6-(trifluoromethyl)pyridine derivatives as potential anti-cancer agents. Bioorganic Medicinal Chemistry Letters2016, 26, 48294831,https://doi.org/10.1016/j.bmcl.2016.08.020.

37. Ashok, D.; Vanaja, B. (2016). Synthesis and anticancer activity evaluation of (E)-3-\{[5-(aryl)-1,3,4oxadiazol-2-yl]methyl \}-5-(3,4,5-trimethoxybenzylidene)thiazolidine-2,4- diones. Journal of General Chemistry2016, 8, 81-685,https://doi.org/10.1134/S1070363216030270.

38. Alzhrani Z.M.M.; Alam, M.M.; Neamatallah, T.; Nazreen, S. Design, synthesis and in vitro antiproliferative activity of new thiazolidinedione-1,3,4-oxadiazole hybrids as thymidylate synthase inhibitors. Journal of Enzyme Inhibition and Medicinal Chemistry2020, 35,11161123,https://doi.org/10.1080/14756366.2020.1759581.

39. Sun, J.; Zhu, H.; Yang, Z.M.; Zhu, H.L. Synthesis, molecular modelling and biological evaluation of 2aminomethyl-5-(quinolin-2-yl)-1,3,4-oxadiazole-2(3H)-thione quinolone derivatives as novel anti-cancer agent. European Journal of Medicinal Chemistry2013, 60 , 28,https://doi.org/10.1016/j.ejmech.2012.11.039.

40. Madhavilatha, B.; Bhattacharjee, D.; Sabitha, G.;Reddy, B.V.S.; Yadav, J.S.; Jain, N.; Reddy, B.J.M. Synthesis and in vitro anti-cancer activity of novel 1,3,4- oxadiazole- linked 1,2,3-triazole/isoxazole hybrids. Journal of Heterocyclic Chemistry2018, 55, 863-870, https://doi.org/10.1002/jhet.3110.

41. Mochona, B.; Mazzio, E.; Gangapurum, M.; Mateeva, N.; Redda, K.K. Synthesis of some benzimidazole derivatives bearing 1,3,4-oxadiazole moiety as anti-cancer agents. Chemical Science Transactions2015, 4, 534-540,https://doi.org/10.7598/cst2015.1029.

42. Ahsan, M.J. Shastri, S. Synthesis of $N$-\{[5-aryl/alkyl-1,3,4-oxadiazol-2-yl]methyl $\}$ pyridin-2-amines as antimicrobial and anti-cancer agents. Electronic Conference on Medicinal Chemistry2015, 9, 118,https://doi.org/10.3390/ecmc-1-A029. 
43. Vinayak, A.; Sudha, M.; Lalita, K. S. Design, Synthesis and characterization of novel amine derivatives of 5[5-(chloromethyl)-1, 3, 4-oxadiazol-2-yl]-2-(4-fluorophenyl)-pyridine as a new class of anti-cancer agents. Dhaka University Journal of Pharmaceutical Sciences2017, 16, 11-19.

44. Ravinaik, B.; Ramachandran, D.; Rao, M.V.B. Synthesis and anti-cancer evaluation of amide derivatives of 1,3,4-oxadiazole linked with benzoxazole. Russian Journal of General Chemistry2019, 89, 10031008,https://doi.org/10.1134/S1070363219050219.

45. Khalil, N.A.; Kamal, A.M.; Emam, S.H. Design, synthesis, and antitumor activity of novel 5-pyridyl1,3,4-oxadiazole derivatives against the breast cancer cell line MCF-7.Biological Pharmaceutical Bulletin2015, 38, 763-773, https://doi.org/10.1248/bpb.b14-00867.

46. Kumar, A.; Gupta, A.; Kashaw, V.; Shukla, G.; Mishra, V.; Kashaw, S.K. Syntheses andanticancer evaluation of some novel 3-[5-(4-substituted) phenyl-1,3,4-oxadiazole-2yl]-2-phenylquinazoline-4(3h)-ones. International Journal of Pharmacy2012, 4, 502-506.

47. Polothi, R.; Raolji, G.S.B.; Kuchibhotla, V.S.; Sheelam, K.; Tuniki, B.; Thodupunuri, P. Synthesis and biological evaluation of 1,2,4-oxadiazole linked 1,3,4-oxadiazole derivatives as tubulin binding agents. Synthetic Communications2019, 49, 1603-1612,https://doi.org/10.1080/00397911.2018.1535076.

48. Hassanzadeh, F.; Sadeghi-Aliabadi, H.; Jaferi, E.; Sharifzadeh, A.; Dana, N. Synthesis and cytotoxic evaluation of some quinazolinone- 5-(4-chlorophenyl) 1,3,4-oxadiazole conjugates. Research in Pharmaceutical Sciences2019, 14, 408-413,https://doi.org/10.4103/1735-5362.268201.

49. Rashid, M.; Husain, A.; Mishra, R. Synthesis of benzimidazoles bearing oxadiazole nucleus as anti-cancer agents. European Journal of Medicinal Chemistry2012, 54, 855866,https://doi.org/10.1016/j.ejmech.2012.04.027.

50. Puthiyapurayil, P.; Poojary, B.; Chikkanna, C.; Buridipad, S.K. Design, synthesis and biological evaluation of a novel series of 1,3,4-oxadiazole bearing $\mathrm{N}$-methyl-4-(trifluoromethyl)phenyl pyrazole moiety as cytotoxic agents. European Journal of Medicinal Chemistry2012, 53, 203210,https://doi.org/10.1016/j.ejmech.2012.03.056.

51. Jisha, M.V.; Kamalabhai, A.V.K.; Babu, G., Biju, C.R. Synthesis, characterization and in vitro anti-cancer screening of novel thiazole- 1,3,4-oxadiazole hybrid analogues. Journal of Chemical and Pharmaceutical Research2013, 5, 64-70.

52. Kapoor, A.; Dhiman, N. Anticancer evaluation of 2-aryl substituted benzimidazole derivatives bearing 1,3,4oxadiazole nucleus. Der Pharma Letters 2016, 8, 149-156.

53. Abdo N.Y.M.; Kamel, M.M. Synthesis and anti-cancer evaluation of 1,3,4-oxadiazoles, 1,3,4-thiadiazoles, 1,2,4-triazoles and mannich bases. Chemistry and Pharmaceutical Bulletin2015, 63, 369376,https://doi.org/10.1248/cpb.c15-00059.

54. Ghag, S P.; Kamath C.R. Synthesis and anti-cancer activity of some novel 1, 3, 4- oxadiazole compounds of 5-amino pyrazole. International Journal of Advance Science Engineering and Technology 2017, 5,52-56.

55. Slawinski, J.; Szafranski, K.; Pogorzelsk, A.; Zolnowska, B.; Macur, K.; Belka, M.; Baczek, T. Novel 2benzylthio-5-(1,3,4-oxadiazol-2-yl) benzene sulfonamide with anticancer activity synthesis, QSAR study and metabolic stability. European Journal of Medicinal Chemistry2017, 132, 236-248, https://doi.org/10.1016/j.ejmech.2017.03.039.

56. Alghamdi, A.A.; Nazreen, S. Synthesis, characterization and cytotoxic study of 2-hydroxy benzothiazole incorporated 1,3,4-oxadiazole derivatives. Egyptian Journal of Chemistry 2020, 63, 471482,https://doi.org/10.21608/EJCHEM.2019.17265.2059.

57. Alam, M.M.; Almalki, A.S.A.; Neamatallah, T.; Ali, N.M.; Malebari, A.M.; Nazreen, S. Synthesis of new 1, 3, 4-oxadiazole-incorporated 1, 2, 3-triazole moieties as potential anti-cancer agents targeting thymidylate synthase and their docking studies. Pharmaceuticals, 2020, 13, 390-405,https://doi.org/10.3390/ph13110390.

58. Alderawy, M.Q.A.; Alrubaie, L.A.R.; Sheri, F.H. Synthesis. Characterization of Ibuprofen N-acyl-1,3,4oxadiazole derivatives and anti-cancer activity against MCF-7 cell line. Systematic Reviews in Pharmacy2020, 11, 681-689.

59. Han,X.; Yu, L.Y.; Ma, D.; Zhang, Z.Y.; Liu, X.H. Synthesis, telomerase in hibitory and anti-cancer activity of new 2-phenyl-4H-chromone derivatives containing 1,3,4-oxadiazole moiety. Journal of Enzyme Inhibition and Medicinal Chemistry2021, 36, 344-360,https://doi.org/10.1080/14756366.2020.1864630.

60. Shanmugam, D.M.K., Rangappa, S., Metri, P.K., Mohan, S., Rangappa, B.K.S Exploring the newer oxadiazoles as real inhibitors of human SIRT2 in hepatocellular cancer cells. Bioorganic and Medicinal Chemistry Letters 2020,30,https://doi.org/10.1016/j.bmcl.2020.127330.

61. Malojirao,V.H.; Girimanchanaika, S.S.; Shanmugam, M.K.; Sherapura, A.; Metri, P.K.; Vigneshwaran, V.; Chinnathambi, A.; Alharbi, S.A.; Rangappa, S.; Mohan, C.D.; Basappa; Prabhakar, B.T.; Rangappa, K.S. Novel 1,3,4-oxadiazole targets STAT3 signaling to induce antitumor effect in lung cancer. Biomedicines, 2020, 8, 368-390,https://doi.org/10.3390/biomedicines8090368.

62. Kumar, P., Rahman, M.A., Wal, P., Rawat, P., Singh, K. Design, synthesis, and anti-cancer evaluation of novel Benzopyran 1, 3, 4-oxadiazole derivatives.Indian J. Heterocyclic Chemistry2020, 30, 395-402.

63. Ono, Y.; Ninomiyaa, M.; Kaneko, D.; Sonawane, A.D.; Udagawa, T.; Tanaka, K.; Nishina, A.; Koketsu, A. Design and synthesis of quinoxaline-1,3,4-oxadiazole hybrid derivatives as potent inhibitors of the anti- 


apoptotic Bcl-2 protein. Bioorganic $\quad$ Chemistry2020, 10245-
10255,https://doi.org/10.1016/j.bioorg.2020.104245.

64. Yahya, T.A.; Abdullah, J.H. Synthesis of some 2,3-dihydro-1,3,4-oxadiazoles and 4,5-dihydro-1,2,4-triazoles as anti-cancer agents. International Journal of Pharmacy and Pharmaceutical Sciences 2020, 12, 9299,http://dx.doi.org/10.22159/ijpps.2020v12i8.36508.

65. Gopinatha, V.K.; Ray, U.; Mantelingu, K.; Raghavan, S,C.; Rangappa, K.S. Synthesis and biological evaluation of theophylline methyl 1,3,4-oxadiazole as anti-cancer agents. Russian Journal of Bioorganic Chemistry 2020, 46, 837-844,https://doi.org/10.1134/S106816202005009X.

66. Popov, S.A.; Semenova, M.D.; Baev, D.S.; Frolova, T.S.; Shestopalov, M.A.; Wang, C.; Qi, Z.; Shults, E.E.; Turks, M. Synthesis and cytotoxicity of hybrids of 1,3,4- or 1,2,5-oxadiazoles tethered from ursane and lupane core with 1,2,3-triazole. Steroids2020, 162, 1-13,https://doi.org/10.1016/j.steroids.2020.108698.

67. Naresh, V.V.; Kumari, Y.B.; Sridhar, M.; Raju, A.D.; Rao, A.S. Synthesis and biological evaluation of 1,3,4oxadiazole fused resveratrol derivatives as anti-cancer agents. Asian Journal of Chemistry, 2020, 32, 19671971,https://doi.org/10.14233/ajchem.2020.22680.

68. Polkam, N.; Malthum, S.; Anireddy, J.S.; Brahma, U.; Vegi, G.M.N. Design, synthesis, and anti-cancer evaluation of new 1,3,4-oxadiazole thioether derivatives. Russian Chemical Bulletin 2021, 70, 580584,https://doi.org/10.1007/s11172-021-3128-0.

69. Abdelrehim, M. S. Synthesis and screening of new $[1,3,4]$ oxadiazole, $[1,2,4]$ triazole, and $[1,2,4]$ triazolo[4,3-b][1,2,4]triazole derivatives as potential antitumor agents on the colon carcinoma cell line (HCT-116). ACS Omega2021, 6, 1687-1696,https://dx.doi.org/10.1021/acsomega.0c05718.

70. Pragathi, Y.J.; Veronica, D.; Rao, M.V.B.; Raju, R.R. Design, synthesis, and anti-cancer activity of 1,3,4oxadiazole incorporated 5-(pyrimidin-5-yl)benzo[d]oxazole derivatives. Russian Journal of General Chemistry, 2020, 90,2371-2375,https://doi.org/10.1134/S1070363220120221.

71. Erensoy, G.; Ding, K.; Zhan, C.; Elmezayen, A.; Yelekci, K.; Duracil, M.; Bingol Ozakpinar, O.; Kukukguzel, I. Synthesis, in silico studies and cytotoxicity evaluation of novel 1,3,4-oxadiazole derivatives designed as potential mPGES-1 inhibitors. Journal of Research in Pharmacy2020, 24, 436451,https://doi.org/10.35333/jrp.2020.187.

72. Cevik, U.A.; Saglik, B.N.; Osmaniye, D.; Levent, S.; Cavusoglu, B.K.; Karaduman, A.B.; Atlid, O.; Eklioglu,O.A.; Kaplancikli, Z.A. Synthesis, anti-cancer evaluation and molecular docking studies of new benzimidazole- 1,3,4-oxadiazole derivatives as human topoisomerase types I poison. Journal of Enzyme Inhibition and Medicinal Chemistry2020, 35, 1657-1673, https://doi.org/10.1080/14756366.2020.1806831. 